Session 1348

\title{
Impact of Applied Research in Engineering Technology
}

\author{
Salahuddin Qazi, Naseem Ishaq \\ State University of New York Institute of Technology \\ P.O. Box 3050, Utica, New York 13504
}

\begin{abstract}
Due to the applied nature of engineering technology programs, it is important that engineering technology faculty stay current to incorporate new technology in their courses and curriculum. It is however difficult for engineering technology faculty to conduct research during the regular semester time because of the hands-on nature of their programs and other college commitments. This paper presents the result of authors' involvement in applied sponsored research, and discusses the impact on the development of courses and curriculum in the department of electrical engineering technology at the State university of New York Institute of Technology at Utica/Rome.
\end{abstract}

\section{INTRODUCTION}

State University of New York (SUNY) Institute of Technology is an upper division transfer college for students who have completed their first two years of higher education at a community college. It also offers graduate programs in accounting, advanced technology, business, computer science and telecommunications.

University research in engineering, according to Lester A. Gerhardt [1] is both a process and a product. It serves to create an environment for engineering education that enhances classroom teaching in terms of relevance and what is taught and how it is taught. A good teacher is often the most active researcher. Further it is the integration of research into teaching which is being adopted by increasing number of educators. It is claimed by these educators that incorporating research examples into their courses and curricula and collaborating with students on research projects result into better research. In addition, the students gain important insight into engineering and science concepts as applied to real life problems [2].

Research is traditionally conducted by the engineering faculty at the universities with graduate programs who can bring research grants and use graduate students to help conduct it. Applied research at institutions offering engineering technology programs is becoming important due to the change in technology and the resulting change in the contents of courses in their programs. It is also important for the faculty to stay current in order to teach effectively and be able to develop new courses in their curriculum. In addition, the business and accrediting agencies are putting pressure on engineering technology programs to make teaching more industry- oriented. Collaborative and joint research between the engineering technology faculty and small businesses can be very beneficial to small businesses in improving their products as they do not have enough funds to spend on research and development. Such collaboration helps the faculty obtain necessary funds and 
equipment at the time of budget cuts and downsizing. The increase in the graduate engineering technology programs in the US is also requiring the faculty to engage in applied research in order to be a responsive teacher.

Conducting applied research by engineering technology faculty, however, is not an easy task because of the applied nature of their program which needs time to set up the labs and conduct the hands-on component of the curriculum. The faculty at the institutions offering engineering technology programs are not expected to hold research credentials for tenure and are not expected to engage in research, and as a result, there is no incentive.

\section{INCORPORATION OF RESEARCH WORK INTO CURRICULUM DEVELOPMENT}

The department of electrical engineering technology is a part of the school of information systems and engineering technology and offers Bachelor's degree programs in electrical engineering technology, computer engineering technology and photonics. The curriculum for the B.S. program in electrical engineering technology includes theoretical issues and emphasizes the use of current,

" state of the art" equipment, and emerging technologies to solve practical design and application problems. This necessitates the development of new courses in emerging technologies and state -ofthe- art equipment and laboratories which is critical because of the strong hands-on emphasis [3]. An interdisciplinary Master of Science program in Advanced Technology with emphasis on practical applications is also jointly offered by the departments of electrical, mechanical and industrial engineering technologies.

To incorporate research into curriculum, the authors actively sought funding for research from both the private and government sectors in the last twelve years. The objective was to undertake applied research and relate it to the development of new courses, updating the existing labs and courses, and involving students in these efforts. Parts of the research project were used as topics for undergraduate student projects which were later incorporated as lab assignments. The results of applied research were also used to develop new courses and labs and updating the existing labs and courses. Listed below are the topics of applied research, the organizations funded the research, and the list of courses it was incorporated to.

\section{Fiber amplifiers and lasers}

This research was conducted at the Rome Lab and was sponsored by the summer faculty research program of the Office of the US Air Force Office of Scientific Research. The research lasted for twelve weeks and was followed by an extension award of $\$ 20,000$ to 25,000 to start research at the author's Institution. The area of research was in fiber amplifiers and lasers. Work involved the development of erbium doped fiber amplifiers and lasers which have revolutionized optical communications at 1550 nanometers wavelength. Work was extended to the development of praseodymium doped fiber amplifiers and lasers which are needed at the commonly used wavelength of 1300 nanometers in optical communications. Some work in the area of mode-locking of fiber doped lasers and use of fiber grating is currently in progress. The authors were able to avail the opportunity of summer faculty programs twice followed by two extension programs to initiate the research at SUNY Institute of Technology. 
The results of the research and equipment obtained was used in updating courses and labs in fiber optics, optical communications and data communications and networking. A new topic on "Industrial applications of optical fiber" was introduced as a part of a graduate course on special topics on advanced technology. Involvement of students in research and spin-off undergraduate projects also resulted in these research projects.

\section{Feature Extraction From Digitized Retinal Images}

This research was sponsored by the State University of New York graduate initiative grant program. It involved interdisciplinary work between the departments of computer science and electrical engineering technology, and a graduate student was hired to help conduct research. Cooperation with a local small business ( PAR Inc.of New Hartford ) resulted in the acquisition of digital retinal images which were used for research. The developed software and hardware were used in the development of a graduate course on computer vision and undergraduate course in image processing and vision.

\section{Eye Access Project}

This research was sponsored by the New York State Science and Technology Foundation in collaboration with a local organization, the Resource Center of Independent Living Inc. of Utica, New York. The work dealt with the investigation and design of an eye tracking system for the use of physically challenged people. It was a joint work with the computer science department, and a graduate student was employed to help develop software. The developed software and hardware was used in an undergraduate course on image processing and vision. A number of projects on feature extractions emerged from this work and were assigned to graduate students resulting in thesis and projects. Based on the research work in sections 2 and 3, a new undergraduate courses "Data compression and Multimedia Technology ", was developed and introduced.

\section{PCs Based Multimedia Communications using Optical Networks}

Work in this area was accomplished by a National Science Foundation sponsored Instrumentation and Laboratory Improvement (ILI) program and college funds. The objective of this work was to link the electrical department's image processing \& computer vision lab with the fiber optic communication lab by an optical network. The enhanced laboratory facilities helped advance instruction for undergraduate students in the areas of fiber optics, data communications \& computer networking, image processing and computer vision, data compression \& multimedia technology, and optical communications [3].

\section{Low Data Rate Multimedia Communications Using Wireless Links}

This research was conducted at the Air Force Lab, Rome, New York, and was sponsored by the summer research faculty program of the US Office of Scientific Research. The duration of the research was 12 weeks and the work involved the areas of commonly used TCP/IP protocols for low data rate using wireless links. A follow on extension grant of $\$ 25,000$ has been applied in the area of Integration of Medium Resource Controller to TCP/IP for Low Data Rate Multimedia 
Communication using wireless links. The result of this research has been used in the development of a new undergraduate course on wireless communication systems.

\section{Use of ADA in Computer Vision}

This work was sponsored by the SUNY Institute of Technology Summer Faculty Research Grant. It involved developing a fast algorithm to process large amount of video data. The results were used in graduate and undergraduate courses on computer vision and image processing.

\section{Flexible Vision Using Coherent Fiber Optics}

This work was supported by SUNY College Foundation for Summer Faculty Research Grant for developing a flexible vision system using coherent fiber optics. The results were applied to the development of a fiber optics and an undergraduate image processing and vision courses and labs.

\section{High Resolution Optical Time Domain Reflectometer}

This work was sponsored by the New York Science and Technology Research Foundation and involved working with a local a manufacturing company (small business) of fiber optic test equipment. The topic dealt with improving the resolution of optical time domain reflectometer using high speed electronic circuitry and helped in the fiber optic and electronics courses.

\section{COURSES AFFECTED}

1. Fiber optics (cross listed with the photonics course).

2. Optical communications (cross listed with the photonics course).

3. Data compression and multimedia communication technology.

4. Data communications and computer networking (cross listed with the computer engineering technology course).

5. Wireless communications systems.

6. Computer and robotic vision ( cross listed graduate course in computer science and advanced technology programs).

7. Special topics in advanced technology (graduate course).

8. Network and multimedia systems ( proposed graduate course).

9. Project (graduate course).

10. Thesis (graduate course in computer science).

\section{CONCLUSION}

A total of ten courses in the area of emerging technology are impacted by incorporating authors' applied research into different aspects of curriculum development. Seven of these courses are taken by the students from undergraduate courses in electrical engineering technology, computer engineering technology, and photonics. Three of the courses are core courses for the graduate programs in advanced technology and taken as electives by the computer science students. Exposure to the new areas of technology and participation in research improved the students' career opportunities and employability. Collaborative research work by faculty and cooperation with local small businesses helped increase the students' technical knowledge relating to the quality and productivity of the operations. This cooperation also helped the college by placing the students for 
employment, internships and arranging field trips to their facilities. It is worth mentioning that the number of small businesses in this area of Oneida County have increased due to the closure and downsizing of high tech industries. Participation of engineering technology faculty in applied research has helped them develop professionally, enrich their curricula and has enhanced the image of the institution at a time when the educational institutions are hit with budget cuts and downsizing. This has also made the engineering technology students more marketable.

\section{ACKNOWLEDGMENT}

The authors would like to acknowledge the US Air Force Office of Scientific Research, the SUNY Institute of Technology and National Science Foundation for sponsoring the research.

\section{REFERENCES}

1. Lester A. Gephardt, "Why University Research ? ". American Society of Engineering Education Prism, PP 14-15, December 1996.

2. Deborah Coppula, " Integrating Teaching and Research ".American Society of Engineering Education Prism, P 19-22, December 1997.

3. Salahuddin Qazi, Naseem Ishaq, "PCs Based Multimedia Communications Systems Usin Optical Networks". Proceeding of Annual Conference of American Society of Engineering Education, Milwaukee WI, June 1997.

\section{BIOGRAPHIES}

SALAHUDDIN QAZI earned a Ph.D. degree in electrical Engineering from Loughborough University, U.K. and is an Associate Professor in the department of electrical engineering technology at the SUNY Institute of Technology, Utica/Rome. His areas of expertise are in the field fiber optics, optical communications and wireless communications. He is currently the coordinator Master of science program in advanced technology which he has helped to develop.

NASEEM ISHAQ earned a Ph.D. degree in electronics engineering from the University of London, U.K. and is an Associate Professor in the department of electrical engineering technology at the State University of New York Institute of technology, Utica/Rome. Her areas of expertise are in the field of image processing \& computer vision, computer aided design, data communications \& multimedia technology. 


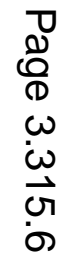

